\title{
Gestión de la responsabilidad social territorial y su relación con la educación superior de calidad
}

\author{
Management of territorial social responsibility and its relationship with quality higher education \\ A gestão da responsabilidade social territorial e sua relação com o ensino superior de qualidade
}

Lilly Huamanchumo Sánchez

lilly.huamanchumo@gmail.com

https://orcid.org/0000-0002-1735-3051

Universidad Nacional Mayor de San Marcos, Lima-Perú

Recibido 6 de abril 2021 | Arbitrado y aceptado 6 de mayo 2021 | Publicado en 01 julio 2021

\section{RESUMEN}

La crisis actual que atraviesa la Universidad y que afecta seriamente su actuación en la sociedad, radica -más allá del papel del Estado-, en el insuficiente quehacer de la formación académica, el limitado trabajo de investigación científica, la inadecuada puesta en marcha de la extensión universitaria, la poca elucidación en torno a lo que significa la responsabilidad social y arraigada en una visión empresarial. El objetivo de la investigación es conocer si la GRST está fuertemente relacionada con la Educación Superior de Calidad (ESC) porque creemos que su implementación en las universidadesdela región, especialmente de Perú, coadyuvaría a que desde un nuevo enfoque de RSU se avance hacia la calidad de la educación superior desde una gestión responsable que la contemple, sin descuidar su rol social. Como conclusión, según Rho de Spearman, La GRST (gestión institucional, formación profesional, investigación, extensión universitaria) y la ESC (actuación, autonomía, crítica, diversidad, equitativa, inclusiva, efectividad, pertinencia) tienen una fuerte y significativa relación a percepción de sus autoridades en las universidades de Perú, 2019, por lo que, si se implementa la GRST en las universidades peruanas, esta tendría éxito gracias a la correspondencia entre ambas variables.

Palabras clave: Universidad; Gestión; Responsabilidad social territorial; Educación Superior; Calidad

\section{ABSTRACT}

The current crisis that the University is going through and that seriously affects its performance in society, lies -beyond the role of the State-, in the insufficient work of academic training, the limited work of scientific research, the inadequate implementation of the university extension, little elucidation about what social responsibility means and rooted in a business vision. The objective of the research is to know if GRST is strongly related to Quality Higher Education (ESC) because we believe that its implementation in universities in the region, especially in Peru, would contribute to advancing from a new approach to USR towards the quality of higher education from a responsible management that contemplates it, without neglecting its social role. In conclusion, according to Rho de Spearman, GRST (institutional management, professional training, research, university extension) and ESC (performance, autonomy, criticism, diversity, equitable, inclusive, effectiveness, relevance) have a strong and significant relationship to perception of its authorities in the universities of Peru, 2019, therefore, if the GRST is implemented in Peruvian universities, it would be successful thanks to the correspondence between both variables.

Key words: University; Management; Territorial social responsibility; Higher education; Quality

\section{RESUMO}

A atual crise que a Universidade atravessa e que afeta seriamente seu desempenho na sociedade, está - além do papel do Estado - na insuficiente formação acadêmica, no limitado trabalho de pesquisa científica, na implementação inadequada da extensão universitária, na falta de esclarecimento do significado da responsabilidade social e enraizada numa visão empresarial. $O$ objetivo da pesquisa é descobrir se o GRST está fortemente relacionado ao Ensino Superior de Qualidade (QHE) porque acreditamos que sua implementação nas universidades da região, especialmente no Peru, contribuiria para uma nova abordagem da USR para avançar em direção à qualidade do ensino superior a partir de uma gestão responsável que o contempla, sem descuidar de seu papel social. Em conclusão, segundo Spearman's Rho, o GRST (gestão institucional, formação profissional, pesquisa, extensão universitária) e o ESC (desempenho, autonomia, crítico, diversidade, equitativo, inclusivo, eficácia, relevância) têm uma forte e significativa relação com a percepção de suas autoridades nas universidades do Peru, 2019, de modo que, se o GRST for implementado nas universidades peruanas, será bem sucedido graças à correspondência entre as duas variáveis.

Palavras-chave:

Universidade; Administração; Responsabilidade social territorial; Educação superior; Qualidade 


\section{INTRODUCCIÓN}

La RS surge con propósitos de justicia, democracia e igualdad para alcanzar finalmente el desarrollo de la sociedad (Rama, 2018). La empresa concibe la RS como vía para resolver los impactos que han generado sus acciones en la sociedad y/o como prevención de conflictos (ISO, 2010; Restrepo, 2013). La academia hace suya la RS con una mirada más humana, en el marco del reconocimiento de la educación superior como medio de cambio social (UNESCO, 1998); por tanto, la RSU puede devolverle la naturaleza social a la universidad (De La Cruz y Sasia, 2008; Delgado, Vargas y Ramos, 2008; Domínguez y Rama, 2012; UNESCO, 2015), de manera que justifique su existencia.

Pero el concepto de la RSU se asumió en muchos países de América Latina, incluso a través de leyes; tal es el caso de Perú (Ley Universitaria $\mathrm{N}^{\circ}$ 30220), con su réplica de la normativa universitaria (UNMSM, 2015) ha sido inadecuado. Además, desde el aporte académico y práctico, esta se planteó con una mirada empresarial, pues redujo su concepción a la gestión de impactos (Vallaeys, 2009), que no es otra cosa que una adaptación de la ISO 26000. Este aporte, si bien es muy importante para la academia, también es insuficiente, según refirió (Grimaldo, 2018). En efecto, la academia como "cuna del saber científico" no puede reducirse a "apagar sus propios incendios". La "casa de la ciencia" debe ser visionaria, solidaria y activa para cumplir con su rol social y atender las diversas demandas sociales largamente postergadas que hoy amenazan incluso la vida del planeta mismo.

Asimismo, Cecchi, Pérez y Sanllorenti (2013) coinciden en señalar que, producto del modelo dominante de la globalización, la Universidad ha descuidado las necesidades sociales, por ello precisan que el Compromiso Social Universitario debe ser visible a través del "trabajo en territorio", entendido como espacio natural de confluencia para la diversidad y como punto de convergencia para superar la conflictividad y con la integración de la docencia, la investigación y la extensión universitaria.

En ese espacio de visible divorcio entre universidad-sociedad, surgela RST (ORSALC, 2016) con una propuesta inspirada en la transformación social desde la acción de diversos frentes, liderados por la academia. El ORSALC1 da vida a la RST y la concibe como una evolución necesaria de la RSU. La RST abandera la lucha contra la injusticia, proclama la libertad y propicia un clima de paz -cimientos indispensables-, para el florecimiento del diálogo y la construcción de un trabajo cooperativo que conduzcan a un cambio social que procure bienestar para todos.

Según se revisó en el marco teórico dela RST, esta se erige en una visión rehumanizadora, estratégica y sostenible (Martin, 2018). Para el estudio, primero es necesario que la academia replantee su papel en la sociedad, se organice, se articule y planifique acciones; es decir, que sea capaz de superar sus propios desafíos institucionales (desafíos internos). De esta manera, podría proyectarse y vincularse

\footnotetext{
1 ORSALC se inauguró en el Palacio de San Carlos, sede de la Cancillería de Colombia avalado por el Ministerio de Relaciones Exteriores y el Ministerio de Educación Nacional de Colombia, en febrero de 2012 (ORSALC, 2018), a cargo del filósofo colombiano Humberto Grimaldo Durán, su coordinador general, convirtiéndose así en una tribuna itinerante en América Latina y el Caribe bajo el paraguas de la RST.

Posteriormente ORSALC fue adscrita al IESALC- UNESCO. Pese a su corta trayectoria de vida ha tenido un importante crecimiento “...Cuenta con una declaración, sedes subregionales, grupos de trabajo, estudios de variada índole, factores e indicadores regionales de análisis y una participación en más de 220 eventos anuales, con una cooperación de 700 instituciones en dos idiomas oficiales (español y portugués)” (Grimaldo, 2018, p.11)
} 
con la sociedad, así como hacerse cargo de los desafíos sociales (desafíos externos) desde una gestión responsable, inteligente y sensible: la GRST (ORSALC, 2016). Es preciso subrayar que el concepto de RST se ha ido edificando y enriqueciendo a la luz del aporte científico-académico de América Latina. Se constituye como un compromiso universitario con el desarrollo social y sostenible que convoca la participación activa de todos los actores sociales, sin distinción alguna.

La RST como propuesta, sistémica y articuladora de procesos, entiende la calidad de la educación superior, como una acción innovadora de la academia en el territorio, abierta a valorar, aprender y respetar los saberes diversos (populares, científicos, internos y externos) para sumar esfuerzos y resolver los problemas que amenazan la sociedad local y mundial y la hacen vulnerable. La declaración CRES 2018, que ve a la educación como un medio para garantizar la libertad e igualdad, pilares de la democracia y desarrollo, se torna viable desde el aporte de la RST para las academias de la región.

En este sentido, la Gestión de Responsabilidad Social Territorial (GRST) propicia la promoción de intercambio de experiencias exitosas a nivel local, nacional y regional, con fines de aprendizaje compartido y de consolidación de alianzas estratégicas que posibiliten la atención de las exigencias actuales de rehumanización, mejoramiento social y protección del ambiente. Así, la GRST debe entenderse como una función estratégica para innovar la educación superior. Para ORSALC (2016), la GRST se concreta en función de cinco componentes: la gestión de impactos, gestión del talento, gestión de la comunicación, gestión de procesos y gestión con personas.

En consecuencia, la crisis actual que atraviesa la Universidad y que afecta seriamente su actuación en la sociedad, radica -más allá del papel del Estado-, en el insuficiente quehacer de la formación académica, el limitado trabajo de investigación científica, la inadecuada puesta en marcha de la extensión universitaria, la poca elucidación en torno a lo que significa la responsabilidad social y arraigada en una visión empresarial.

De esta forma, alteradas y desarticuladas las tres funciones principales de la Universidad y con la ausencia de una gestión responsable e inteligente que vincule y viabilice sus funciones prioritarias en procesos efectivos, la tarea social de la academia se vio seriamente desconfigurada, afectando su rol social que por naturaleza le corresponde cumplir. El objetivo de la investigación es conocer si la GRST está fuertemente relacionada con la Educación Superior de Calidad (ESC) porque se cree que su implementación en las universidades de la región, especialmente de Perú, coadyuvaría a que desde un nuevo enfoque de RSU se avance hacia la calidad de la educación superior desde una gestión responsable que la contemple, sin descuidar su rol social.

\section{MÉTODO}

Luego de realizar el análisis de fiabilidad de los dos cuestionarios para cada variable (GRST y ESC), se depuraron algunos ítems para poder alcanzar un valor apropiado del estadístico Alfa de Cronbach. Para el instrumento que mide la Gestión de Responsabilidad Social Territorial, se obtuvo un Alfa de Cronbach igual a 0.878, que indica un aceptable grado de confiabilidad. De los 62 ítems quedaron finalmente 50 ítems. Para el instrumento que mide la Educación Superior de Calidad, se obtuvo un Alfa de Cronbach igual a 0.902, que indica un alto grado de confiabilidad. De los 35 ítems iniciales, quedaron finalmente 32 ítems.

\section{RESULTADOS Y DISCUSIÓN}

Conforme a los resultados de la prueba de la hipótesis general, se demostró que existe una relación altamente significativa entre la GRST y 
la ESC en la percepción de sus autoridades de las universidades del Perú, 2019. Así, la GRST fue desarrollada a partir de cuatro dimensiones: gestión institucional, formación académica, investigación y extensión universitaria (RSU-RUS); esto, por considerar la educación de calidad como una vía de transformación de la persona y de la sociedad (ORSALC, 2016; Grimaldo, 2018; Martin, 2018). Ello se relaciona con las ocho dimensiones de la ESC: actuación, autonomía, crítica, diversidad, equitativa, inclusiva, efectiva y pertinencia, pues, según lo referido en el trabajo, estas facilitan su concreción. Por otro lado, también aportan a resolver las tensiones de alta conflictividad actual y futura que podrían afectar la educación superior, según se anticipó en el informe Delors (Delors et al, 1996).

\section{Variable: Gestión de Responsabilidad Social Territorial}

\section{Dimensión. Gestión Institucional}

En la Tabla 1, la dimensión gestión institucional (gestión de impactos, gestión del talento, gestión de la comunicación, gestión de procesos y gestión con personas) presenta valores de percepción que alcanzan una media de 99,63, cercana al valor máximo 132 y lejana del valor mínimo 33, lo que indica un buen desempeño en este aspecto. El coeficiente de variación (18 \%) señala un comportamiento uniforme, lo cual apunta a la homogeneidad de las respuestas.

Tabla 1. Estadísticos descriptivos: resumen dimensión gestión institucional.

\begin{tabular}{cccccc}
\hline & $\mathbf{N}^{\circ}$ & Mínimo & Máximo & Media & Desviación estándar \\
\hline Gestión Institucional & 142 & 33 & 132 & 99,63 & 17,555 \\
\hline
\end{tabular}

En la Figura 1, se muestra el resumen de la gestión institucional, que presenta 27 ítems con cinco valores de percepción (1: totalmente en desacuerdo; 2: en desacuerdo; 3: ni en desacuerdo ni de acuerdo; 4: de acuerdo; y 5: totalmente de acuerdo). Finalmente, se muestra un total de 135 indicadores de tendencia (27 ítems x 5 valores de percepción) para esta dimensión, cuya frecuencia de mayor alcance llega a 89 encuestados (82-108) que están de acuerdo; 36 encuestados, totalmente de acuerdo (109-133); y 13 encuestados (54-81), ni en desacuerdo ni de acuerdo. Por otro lado, 4 encuestados están en desacuerdo (29-53), y ninguno está totalmente en desacuerdo (0). El gráfico ilustra un comportamiento más o menos simétrico de esta variable. 


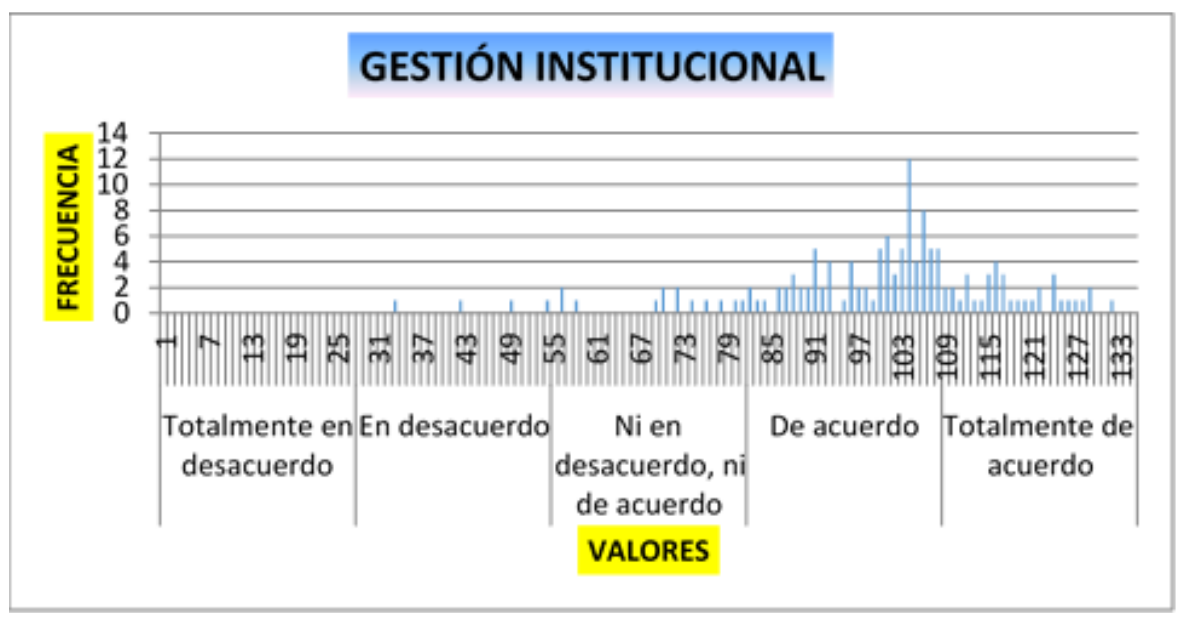

Figura 1. Resumen dimensión: gestión institucional.

\section{Dimensión. Gestión de la formación académica}

Del mismo modo, en la Tabla 2, la dimensión gestión de la formación académica (gestión curricular y plan de estudios, evaluación curricular, innovación) presenta valores de percepción que alcanzan una media de 40,40, cercana al valor máximo 55 ylejana del valor mínimo 16, lo que indica un buen desempeño en este aspecto. El coeficiente de variación (19\%) apunta a un comportamiento uniforme, lo cual implicó la homogeneidad de las respuestas.

Tabla 2. Estadísticos descriptivos: resumen dimensión gestión de la formación académica.

\begin{tabular}{ccccccc}
\hline & $\mathbf{N}^{\circ}$ & Mínimo & Máximo & Media & $\begin{array}{c}\text { Desviación } \\
\text { estándar }\end{array}$ & CV \\
\hline $\begin{array}{c}\text { Gestión de la formación } \\
\text { académica }\end{array}$ & 142 & 16 & 55 & 40,40 & 7,647 & $19 \%$ \\
\hline
\end{tabular}

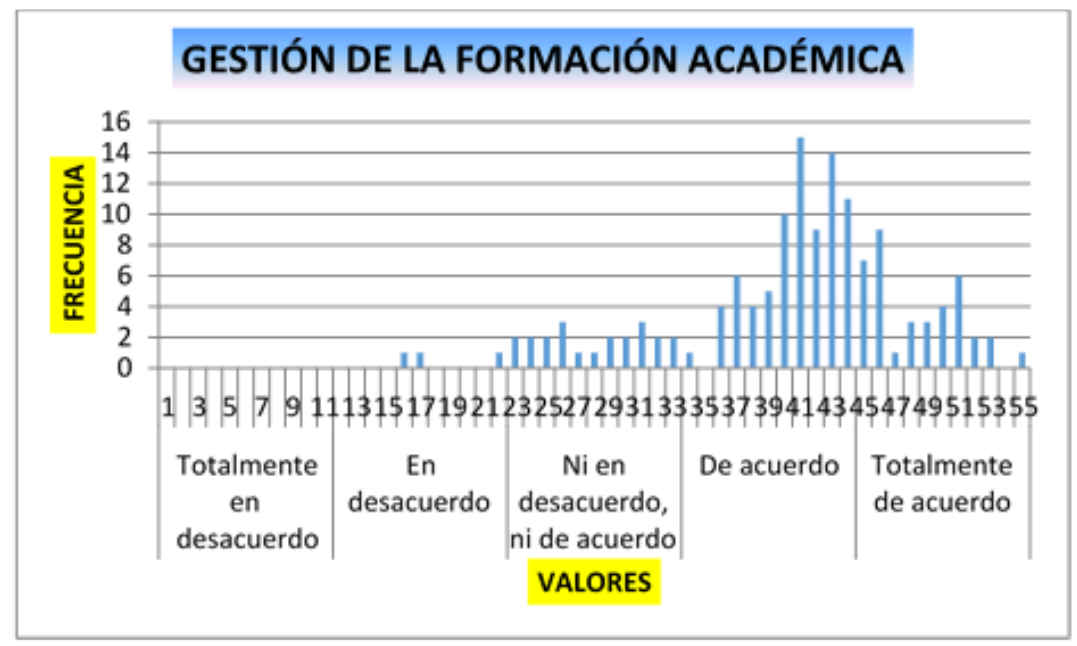

Figura 2. Resumen dimensión gestión de la formación académica. 
Dimensión. Gestión de la Investigación

En la Tabla 3, la dimensión gestión de la investigación (gestión de la investigación) presenta valores de percepción que alcanzan una media de 18,54, cercana al valor máximo 25 y lejana del valor mínimo 6, lo que indica un buen desempeño en este aspecto. El coeficiente de variación (20 \%) apunta a un comportamiento uniforme, lo cual muestra la homogeneidad de las respuestas.

Tabla 3. Estadísticos descriptivos: resumen, dimensión gestión de la investigación.

\begin{tabular}{ccccccc}
\hline & $\mathbf{N}^{\circ}$ & Mínimo & Máximo & Media & $\begin{array}{c}\text { Desviación } \\
\text { estándar }\end{array}$ & CV \\
\hline Gestión de la investigación & 142 & 6 & 25 & 18,54 & 3,749 & $20 \%$ \\
\hline
\end{tabular}

\section{Dimensión. Gestión de la Extensión Universitaria}

El consolidado de la dimensión gestión de la extensión universitaria se muestra en la Tabla 4.

Tabla 4. Estadísticos descriptivos: resumen dimensión gestión de la extensión universitaria.

\begin{tabular}{ccccccc}
\hline & $\mathbf{N}^{\circ}$ & Mínimo & Máximo & Media & $\begin{array}{c}\text { Desviación } \\
\text { estándar }\end{array}$ & CV \\
\hline $\begin{array}{c}\text { Gestión de la extensión } \\
\text { universitaria }\end{array}$ & 142 & 8 & 35 & 25,80 & 6,345 & $25 \%$ \\
\hline
\end{tabular}

En la Tabla 4, se observa el resumen de la dimensión gestión de la extensión universitaria, el cual presenta valores de percepción que alcanzan una media de 25,80, cercana al valor máximo 35 y lejana del valor mínimo 8 , lo que indica un buen desempeño en este aspecto. El coeficiente de variación $(25 \%)$ apunta a un comportamiento homogéneo, lo cual demuestra la homogeneidad de las respuestas. Se observa el sesgo hacia la derecha, hacia los valores más altos.

\section{Variable: Educación superior de calidad}

\section{Dimensión. Actuación}

En la Tabla 5, se relaciona el resumen de la dimensión actuación, la cual presenta valores de percepción que alcanzan una media de 29,99, cercana al valor máximo 40 y lejana del valor mínimo 12, lo que indica un buen desempeño en este aspecto. El coeficiente de variación (23\%) implica un comportamiento uniforme, que apunta a la homogeneidad de las respuestas.

Tabla 5. Estadísticos descriptivos: resumen dimensión actuación.

\begin{tabular}{ccccccc}
\hline & $\mathbf{N}^{\circ}$ & Mínimo & Máximo & Media & $\begin{array}{c}\text { Desviación } \\
\text { estándar }\end{array}$ & CV \\
\hline Actuación & 142 & 12 & 40 & 29,99 & 6,769 & $23 \%$ \\
\hline
\end{tabular}


En la Figura 3, se muestra que la dimensión actuación presenta cinco ítems con cinco valores de percepción (1: totalmente en desacuerdo; 2 : en desacuerdo; 3: ni en desacuerdo ni de acuerdo; 4: de acuerdo; y 5: totalmente de acuerdo). Finalmente, se señala un total de 8 indicadores de tendencia para esta dimensión, cuya frecuencia de mayor alcance llega a 76 encuestados (13-18) que están de acuerdo; 4 encuestados, totalmente de acuerdo (25-30); y 52 encuestados (13-18), ni en desacuerdo ni de acuerdo. Por otro lado, hay 9 encuestados que están en desacuerdo (7-12), y 1 está totalmente en desacuerdo (0-6). En el gráfico se observa el sesgo de los datos hacia la parte derecha, con los valores más altos respecto al lado izquierdo.

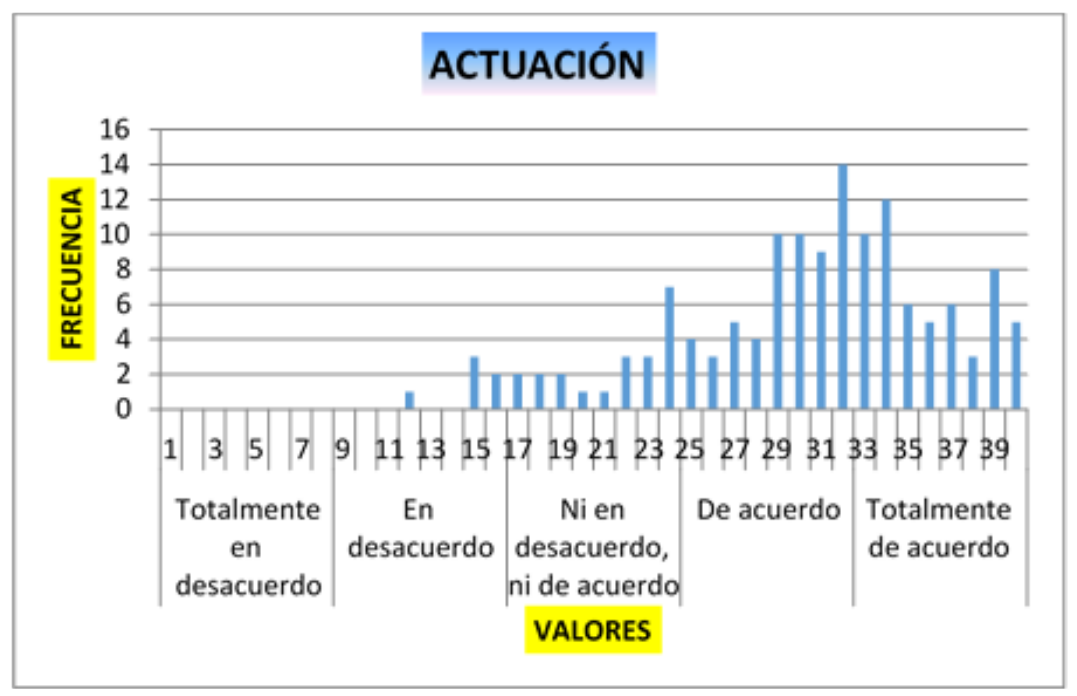

Figura 3. Dimensión actuación.

\section{Dimensión. Autonomía}

En la Tabla 6 se detalla la dimensión de autonomía de la variable Educación Superior de calidad.

Tabla 6. Estadísticos descriptivos: resumen dimensión autonomía.

\begin{tabular}{ccccccc}
\hline & $\mathbf{N}^{\circ}$ & Mínimo & Máximo & Media & $\begin{array}{c}\text { Desviación } \\
\text { estándar }\end{array}$ & CV \\
\hline Autonomía & 142 & 4 & 20 & 15,54 & 3,827 & $25 \%$ \\
\hline
\end{tabular}

El valor promedio de la dimensión autonomía de la ESC es más cercano al valor máximo 20 y más lejano del valor mínimo 4; esto denota el buen desempeño de dicha dimensión. Igualmente, la media es de 15,54 y el coeficiente de variación es del $25 \%$, lo que apunta a la homogeneidad de los datos. Un sesgo hacia los valores más altos se ilustra en la Figura 4. 


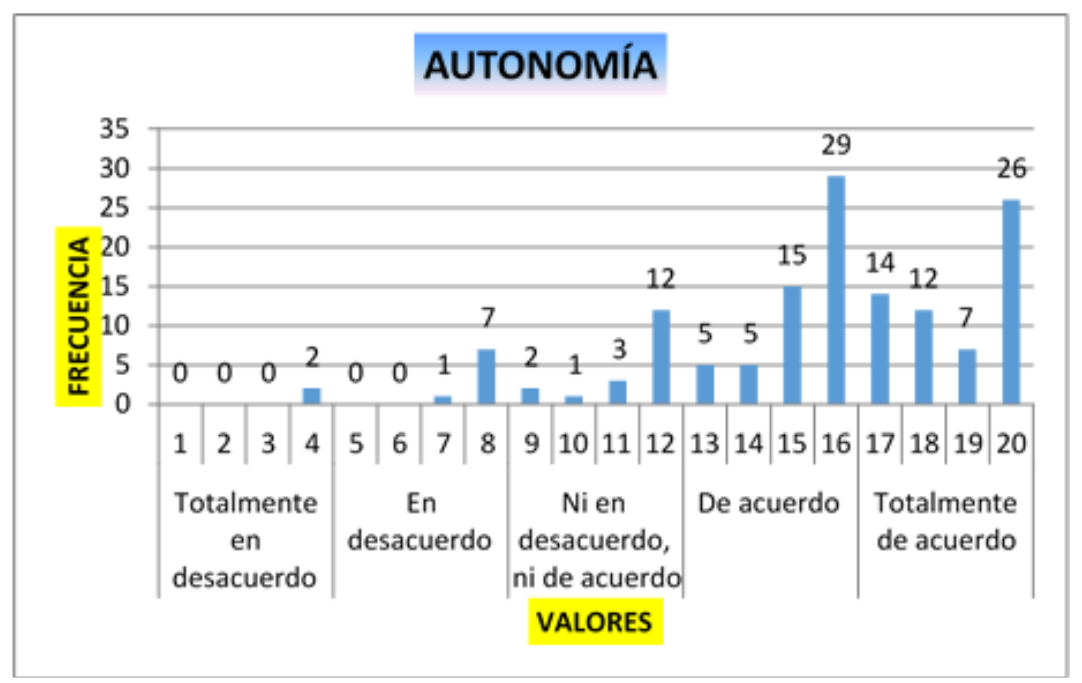

Figura 4. Dimensión autonomía.

En la Figura 4, se muestra que la dimensión autonomía presenta cuatro ítems con cinco valores de percepción (1: totalmente en desacuerdo; 2 : en desacuerdo; 3: ni en desacuerdo ni de acuerdo; 4: de acuerdo; y 5: totalmente de acuerdo). Finalmente, se relaciona un total de 20 indicadores de tendencia para esta dimensión, cuya frecuencia de mayor alcance llega a 76 encuestados (13-18) que están de acuerdo; 4 encuestados, totalmente de acuerdo (25-30); y 52 encuestados (13-18), ni en desacuerdo ni de acuerdo. Por otro lado, 9 encuestados están en desacuerdo (7-12), y 1 está totalmente en desacuerdo (0-6). En el gráfico se observa el sesgo de los datos hacia la parte derecha, con los valores más altos respecto al lado izquierdo.

\section{Dimensión. Diversidad}

Los resultados encontrados sobre la dimensión diversidad se observa en la Tabla 7 . Para el consolidado de la dimensión diversidad de la ESC, el valor promedio es cercano al valor máximo 10 y lejano del mínimo 3; ello denota un buen comportamiento de la variable. El coeficiente de variación (23\%) también indica una homogeneidad en los datos.

Tabla 7. Estadísticos descriptivos: resumen dimensión diversidad.

\begin{tabular}{ccccccc}
\hline & $\mathbf{N}^{\circ}$ & Mínimo & Máximo & Media & $\begin{array}{c}\text { Desviación } \\
\text { estándar }\end{array}$ \\
\hline Diversidad & 142 & 3 & 10 & 7,89 & $1,834 \quad 23 \%$ \\
\hline
\end{tabular}




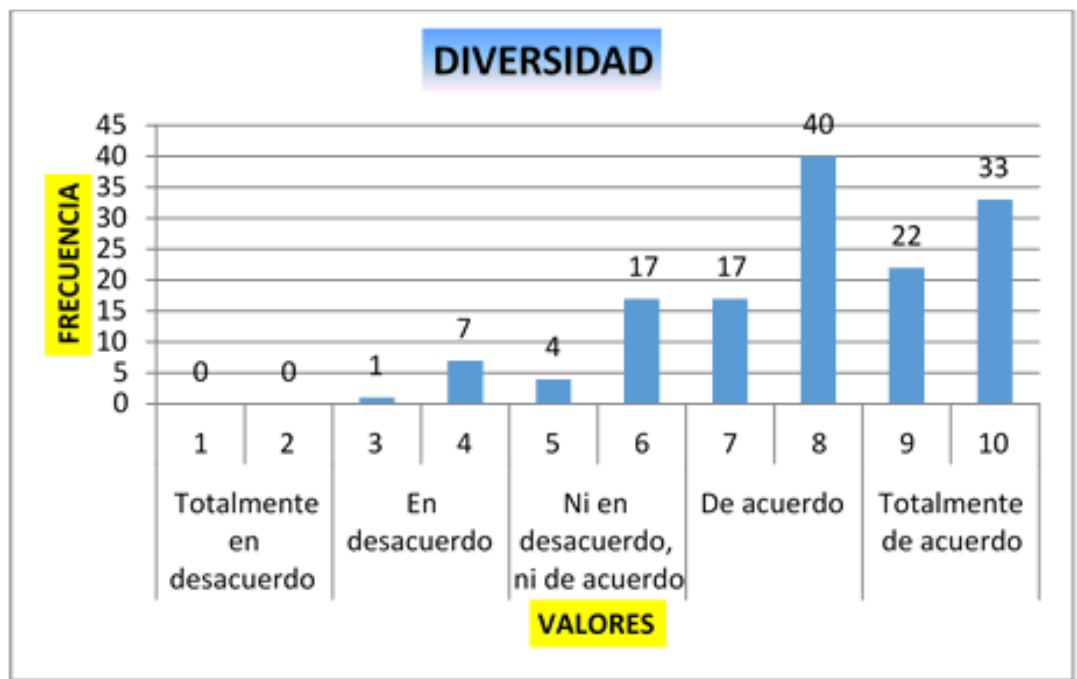

Figura 5. Dimensión diversidad.

En la Figura 5, se muestra que la dimensión diversidad presenta dos ítems con cinco valores de percepción (1: totalmente en desacuerdo; 2: en desacuerdo; 3: ni en desacuerdo ni de acuerdo; 4: de acuerdo; y 5: totalmente de acuerdo). Finalmente, se relaciona un total de 10 indicadores de tendencia para esta dimensión, cuya frecuencia de mayor alcance llega a 76 encuestados (13-18) que están de acuerdo; 4 encuestados, totalmente de acuerdo (25-30); y 52 encuestados (13-18), ni en desacuerdo ni de acuerdo. Por otro lado, 9 encuestados están en desacuerdo (7-12), y 1 está totalmente en desacuerdo (0-6). En el gráfico se observa el sesgo de los datos hacia la parte derecha, con los valores más altos respecto al lado izquierdo.

\section{Dimensión. Equidad}

Para el consolidado de la dimensión equidad, el valor promedio es bastante cercano al valor máximo 10 y lejano del mínimo 2, lo que denota un buen comportamiento de la variable. El coeficiente de variación (26\%) indica una homogeneidad en los datos. Esto se puede visualizar en la Tabla 8.

Tabla 8. Estadísticos descriptivos: resumen dimensión equidad.

\begin{tabular}{ccccccc}
\hline & $\mathbf{N}^{\circ}$ & Mínimo & Máximo & Media & $\begin{array}{c}\text { Desviación } \\
\text { estándar }\end{array}$ \\
\hline Equidad & 142 & 2 & 10 & 7,92 & 2,068 & $26 \%$ \\
\hline
\end{tabular}




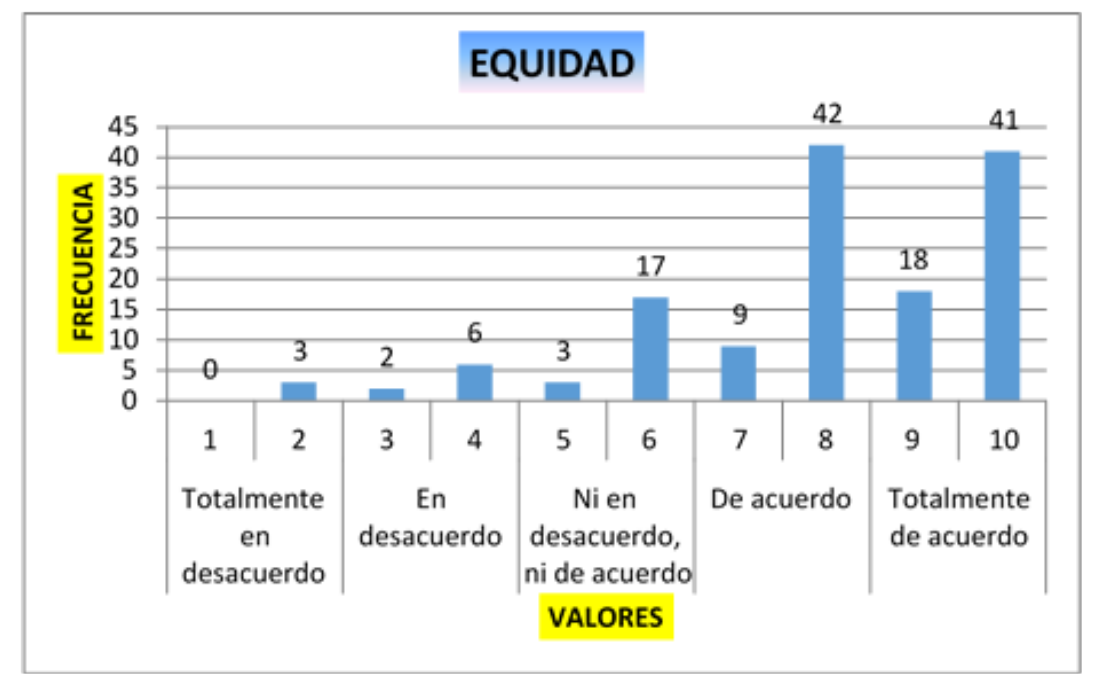

Figura 6. Dimensión equidad.

En la Figura 6, se muestra que la dimensión diversidad presenta dos ítems con cinco valores de percepción (1: totalmente en desacuerdo; 2 : en desacuerdo; 3: ni en desacuerdo ni de acuerdo; 4: de acuerdo; y 5: totalmente de acuerdo). Finalmente, se halla un total de 10 indicadores de tendencia para esta dimensión, cuya frecuencia de mayor alcance llega a 76 encuestados (13-18) que están de acuerdo; 4 encuestados, totalmente de acuerdo (25-30); y 52 encuestados (13-18), ni en desacuerdo ni de acuerdo. Por otro lado, 9 encuestados están en desacuerdo (7-12), y 1 está totalmente en desacuerdo (0-6). En el gráfico se observa el sesgo de los datos hacia la parte derecha, con los valores más altos respecto al lado izquierdo.

\section{Dimensión. Inclusiva}

En la Tabla 9 se detalla los resultados de la dimensión inclusiva de la Educación Superior de calidad. En el consolidado de la dimensión inclusiva, el promedio se acerca bastante al valor máximo 15 y se ve lejano del valor mínimo 3, lo que denota el buen desempeño de la variable. El coeficiente de variación (25\%) indica la homogeneidad de los datos.

Tabla 9. Estadísticos descriptivos: resumen dimensión inclusiva.

\begin{tabular}{lccccc}
\hline & $\mathbf{N}^{\circ}$ & Mínimo & Máximo & $\begin{array}{c}\text { Desviación } \\
\text { estándar }\end{array}$ \\
\hline Inclusiva & 142 & 3 & 15 & 11,86 & 2,974 \\
\hline
\end{tabular}




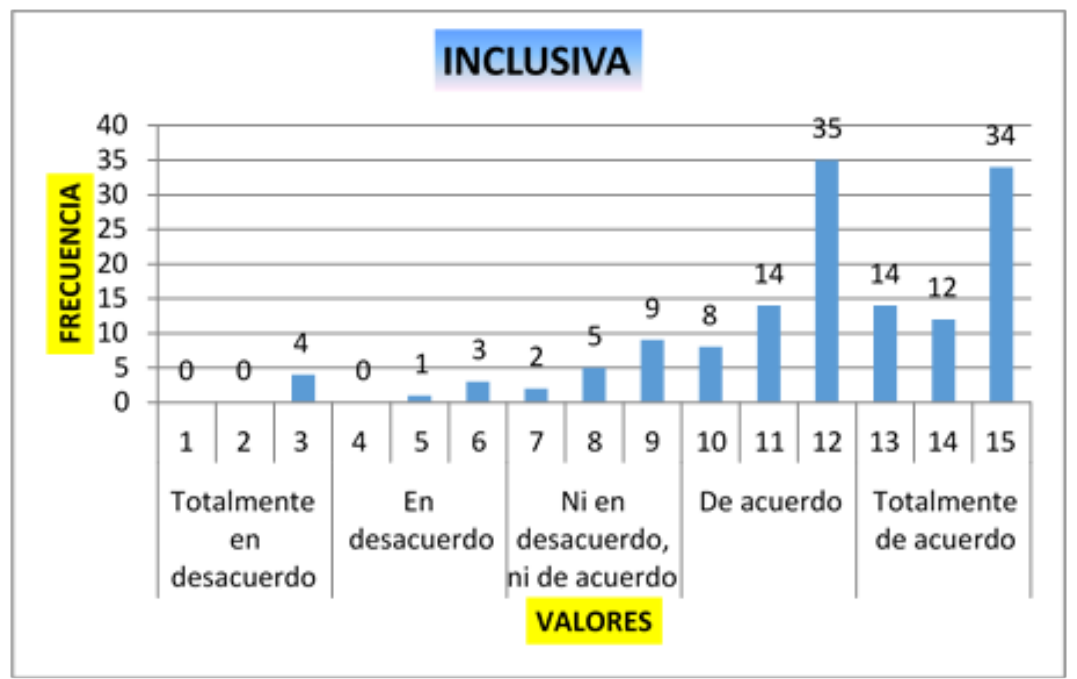

Figura 7. Dimensión inclusiva.

En la Figura 7, se muestra que la dimensión inclusiva presenta tres ítems con cinco valores de percepción (1: totalmente en desacuerdo; 2: en desacuerdo; 3: ni en desacuerdo ni de acuerdo; 4: de acuerdo; y 5: totalmente de acuerdo). Finalmente, se relaciona un total de 15 indicadores de tendencia para esta dimensión, cuya frecuencia de mayor alcance llega a 76 encuestados (13-18) que están de acuerdo; 4 encuestados, totalmente de acuerdo (25-30); y 52 encuestados (13-18), ni en desacuerdo, ni de acuerdo. Por otro lado, 9 encuestados están en desacuerdo (7-12), y 1 está totalmente en desacuerdo (0-6). En el gráfico se observa el sesgo de los datos hacia la parte derecha, con los valores más altos respecto al lado izquierdo.

\section{Dimensión. Efectividad}

En el consolidado de la dimensión efectividad, el promedio se acerca bastante al valor máximo 45 y se encuentra lejano del mínimo 11, lo que denota el buen desempeño de esta variable. El coeficiente de variación $(24 \%)$ indica la homogeneidad de los datos. Esto se puede detallar en la Tabla 10.

Tabla 10. Estadísticos descriptivos: resumen dimensión efectividad.

\begin{tabular}{ccccccc}
\hline & N $^{\circ}$ & Mínimo & Máximo & Media & $\begin{array}{c}\text { Desviación } \\
\text { estándar }\end{array}$ & CV \\
\hline Efectividad & 142 & 11 & 45 & 35,56 & 8,564 & $24 \%$ \\
\hline
\end{tabular}




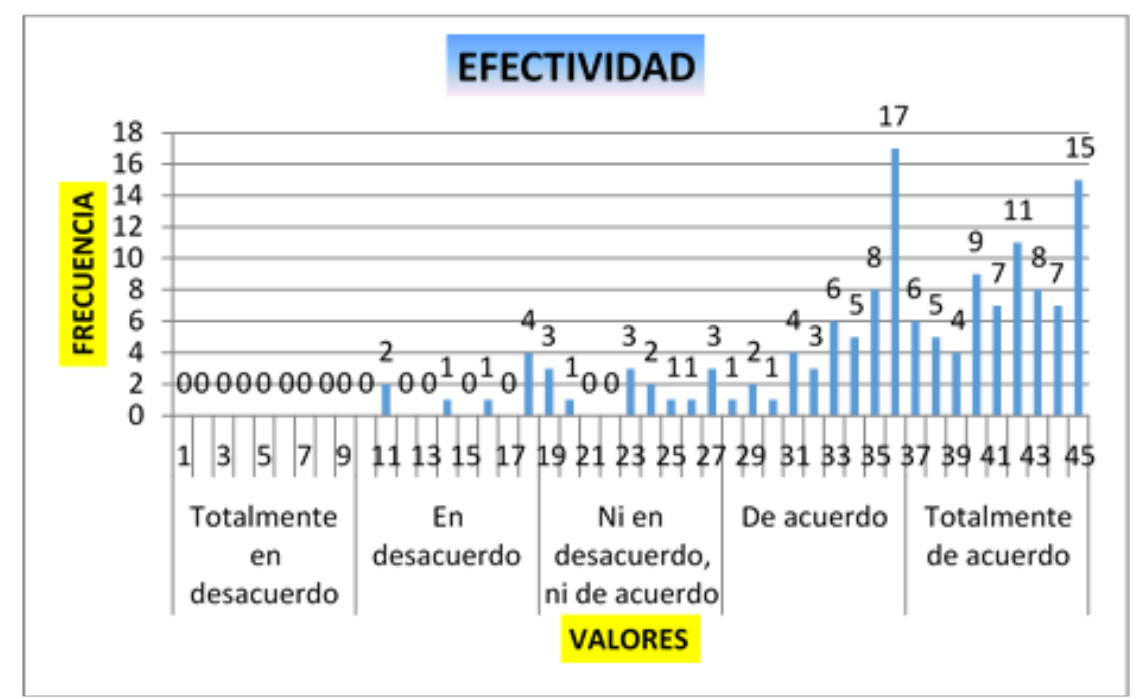

Figura 8. Dimensión efectividad.

En la Figura 8, se muestra que la dimensión inclusiva presenta tres ítems con cinco valores de percepción (1: totalmente en desacuerdo; 2: en desacuerdo; 3: ni en desacuerdo ni de acuerdo; 4: de acuerdo; y 5: totalmente de acuerdo). Finalmente, se relaciona un total de 15 indicadores de tendencia para esta dimensión, cuya frecuencia de mayor alcance llega a 76 encuestados (13-18) que están de acuerdo; 4 encuestados, totalmente de acuerdo (25-30); y 52 encuestados (13-18), ni en desacuerdo ni de acuerdo. Por otro lado, 9 encuestados están en desacuerdo (7-12), y uno está totalmente en desacuerdo (0-6). En el gráfico se observa el sesgo de los datos hacia la parte derecha, con los valores más altos respecto al lado izquierdo.

\section{Dimensión. Pertinencia}

El consolidado de la dimensión pertinencia, en su valor promedio, se acerca al valor máximo 10 y se aleja del mínimo 2, lo que señala el buen desempeño de la variable. El coeficiente de variación indica un comportamiento homogéneo. Esto se observa en la Tabla 11.

Tabla 11. Estadísticos descriptivos: resumen dimensión pertinencia.

\begin{tabular}{ccccccc}
\hline & $\mathbf{N}^{\circ}$ & Mínimo & Máximo & Media & $\begin{array}{c}\text { Desviación } \\
\text { estándar }\end{array}$ & CV \\
\hline Pertinencia & 142 & 2 & 10 & 7,91 & 2,063 & $26 \%$ \\
\hline
\end{tabular}




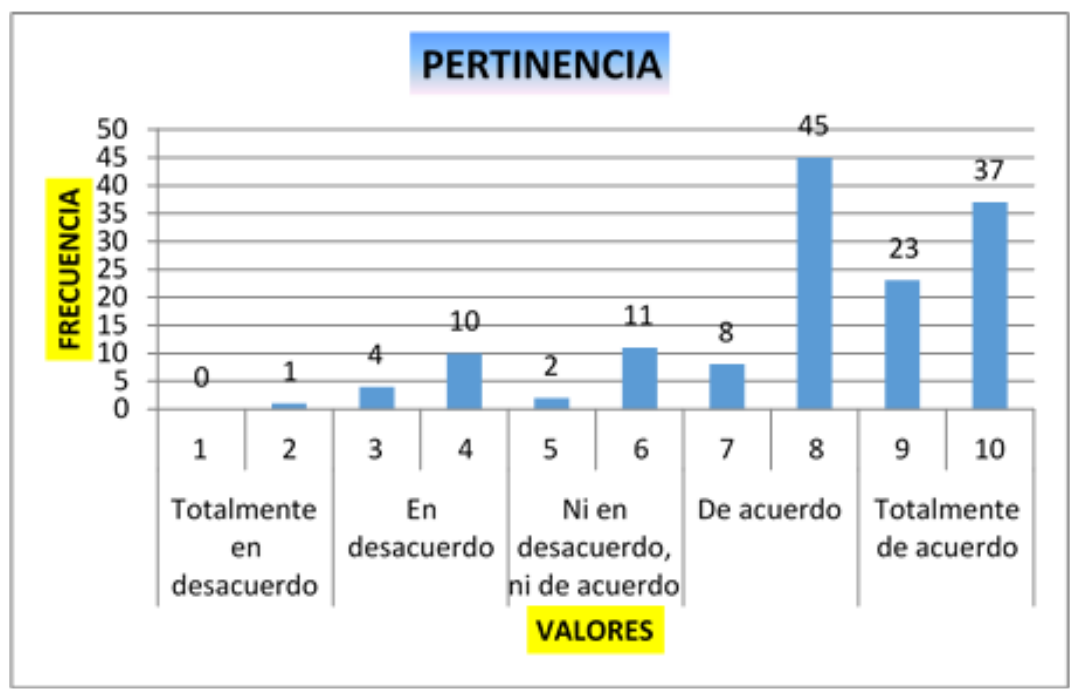

Figura 9. Dimensión pertinencia.

En la Figura 9, se muestra que la dimensión inclusiva presenta tres ítems con cinco valores de percepción (1: totalmente en desacuerdo; 2: en desacuerdo; 3: ni en desacuerdo ni de acuerdo; 4: de acuerdo; y 5: totalmente de acuerdo). Finalmente, se prueba un total de 15 indicadores de tendencia para esta dimensión, cuya frecuencia de mayor alcance llega a 76 encuestados (13-18) que están de acuerdo; 4 encuestados, totalmente de acuerdo (25-30); y 52 encuestados (13-18), ni en desacuerdo ni de acuerdo. Por otro lado, hay 9 encuestados que están en desacuerdo (7-12), y 1 que está totalmente en desacuerdo (0-6). En el gráfico se observa el sesgo de los datos hacia la parte derecha, con los valores más altos respecto al lado izquierdo.

\section{Prueba de hipótesis}

Para la verificación de las hipótesis de investigación en la presente tesis se consideraron un nivel de confianza del $95 \%$ y la siguiente regla de verificación estadística: si la significancia empírica (valor p) es menor que la significancia teórica (5\%), se rechaza la hipótesis nula (Ho).

Existe una relación altamente significativa entre la GRST y la ESC en la percepción de sus autoridades de las universidades de Perú, 2019.

Ho: no existe correlación entre ambas variables.

H1: existe correlación entre ambas variables.

En la Tabla 12, se detalla el cálculo de la correlación Rho de Spearman para la hipótesis general que involucra las variables de estudio. 
Tabla 12. Correlaciones GRST y ESC.

\begin{tabular}{lllcc}
\hline & & GRST & ESC \\
\hline \multirow{2}{*}{ GRST } & Coeficiente de correlación & Valor p & 1,000 & $0,757^{* *}$ \\
& & $\mathrm{~N}^{\circ}$ & $\cdot$ &, 000 \\
\multirow{3}{*}{ Rho de Spearman } & & Coeficiente de correlación & 142 & 142 \\
& \multirow{2}{*}{ ESC } & Valor p & $0,757^{* *}$ & 1,000 \\
& & $\mathrm{~N}^{\circ}$ &, 000 &. \\
& & & 142 & 142 \\
\hline
\end{tabular}

El resultado fue de 0,757 , lo que indica una correlación positiva regular. Es decir, si mejora la GRST, mejora la ESC. Por otra parte, el valor p obtenido $(0,000)$ es menor que la significancia teórica (5\%), por lo que se rechaza la Ho y se verifica la hipótesis general. La Figura 10 representa el diagrama de dispersión que ilustra la relación positiva o directa entre ambas variables.

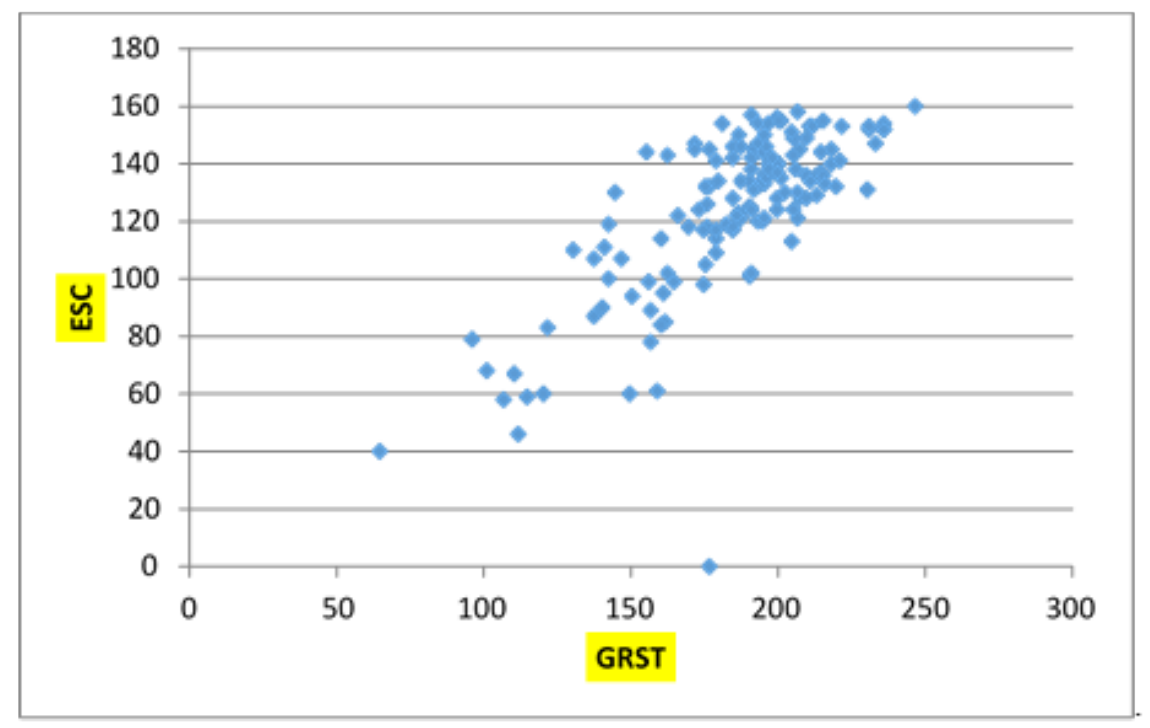

Figura 10. Dispersión GRST/ESC.

El resultado entre las dos variables GRST Y ESC fue de 0,791, lo que implica una correlación positiva regular. Con esto se entiende que, si se implementa la GRST en la universidad, ha de mejorar la calidad de la educación superior. La comprobación de la hipótesis general es un excelente resultado, teniendo en cuenta que las personas consultadas a través de los dos instrumentos sobre GRST y ESC fueron los directores de responsabilidad social y jefes de calidad de las universidades peruanas; es decir, las personas más idóneas para aportar información desde el conocimiento y la experiencia.

Como se vio en el marco teórico, existen diversas concepciones sobre la calidad de la educación en América Latina y el Caribe. De todas 
las propuestas plasmadas en la investigación, se destacan tres de ellas que, por su relevancia y complementariedad, se consideran oportunas; esto, atendiendo la coyuntura y con miras a lograr un futuro promisorio. En primer lugar, es importante la calidad como proceso, porque valora los logros del estudiante a lo largo del recorrido de su formación (Cobo, 1995, como se citó en Valdés, 2008), y porque se compromete en formar ciudadanos competentes profesionalmente y con sólidos valores personales (Martin, 2018).

Finalmente, la calidad como acción para la transformación (UNESCO, 2019; UNESCO, 2015; CRES, 2018; ORSALC, 2016; Grimaldo, 2018; Martin, 2018) asume el proceso enseñanzaaprendizaje más allá de las aulas y convoca a los estudiantes a involucrarse con la realidad, así como a los docentes capaces de innovar prácticas educativas a través de metodologías adecuadas que se concretan en el territorio, como el aprendizaje de servicio. Se considera que, tanto en la teoría como en la práctica, las dos primeras concepciones se han asumido progresivamente en la región y en el Perú, siempre con el fin de perfeccionar aspectos relacionados con su medición. Sin embargo, la última, que ha sido denominada actuación para la transformación debe asumirse con decisión, se debe pasar de la teoría a la acción, más allá del aula universitaria y en contacto pleno con la realidad doliente (ORSALC, 2015).

En pleno siglo XXI, en el marco de los ODS 2030 y frente a una serie de desafíos sociales, la educación de calidad debe comprenderse como aquella que viabiliza dicha transformación, la cual se inicia con la formación de un ciudadano comprometido con sus deberes y derechos. Lamentablemente, la historia de las últimas décadas de América Latina y el Caribe se caracteriza por prácticas que develan injusticia, indiferencia y corrupción, entre otros flagelos que conducen a la deshumanización y la barbarización frente a la escasez de valores. Ello obliga a analizar y ajustar los procesos de formación que desarrollan las academias, pues más allá de lo profesional, se trata de rehumanizar; de lo contrario, la empatía, la solidaridad y el dolor de los más vulnerables siempre han de ser postergados. En ese sentido, se destaca que el perfil del docente del corriente siglo debe contar con algunas cualidades: innovador, sensible, ético, empático, responsable, gestor, líder, investigador, conocedor de las Tecnologías de la Información y la Comunicación (TIC), estratega y, fundamentalmente, maestro desde el ejemplo.

\section{CONCLUSIONES}

La GRST (gestión institucional, formación profesional, investigación, extensión universitaria) y la ESC (actuación, autonomía, crítica, diversidad, equitativa, inclusiva, efectividad, pertinencia) tienen una fuerte y significativa relación a percepción de sus autoridades en las universidades de Perú, 2019. Así las cosas, si se implementa la GRST en las universidades peruanas, esta tendría éxito gracias a la correspondencia entre ambas variables.

Asimismo, las autoridades de responsabilidad social y de calidad consideran altamente efectivas los subdimensiones de la gestión institucional, por lo que su aplicación para consolidar una gestión de calidad resulta idónea. La gestión de la formación académica que contempla la GRST debe expandir sus horizontes, superar el claustro universitario y concretarse en interrelación con las comunidades del territorio, lo cual coadyuvaría a sensibilizar a los estudiantes y comprometerlos en la acción participante y crítica de su entorno.

Del mismo modo, la gestión de la investigación que contempla la GRST deja traslucir la actual 
desvinculación con ESC; por lo que, su aplicación contribuiría a dar soluciones a los problemas sociales desde la investigación, de forma que estos se consoliden en el marco de los ODS 2030. Y finalmente, la gestión de la extensión universitaria (RSU-RUS) que contempla la GRST permite que la RST tenga un enfoque de la RSU que se concreta en las demandas y necesidades del territorio. Asumir la RST y la GRST en la academia posibilitaría que la tercera función sustantiva devolviera a la academia su rol social.

\section{REFERENCIAS}

Cecchi, N., Pérez, D., y Sanllorenti, P. (2013). Compromiso social universitario. De la universidad posible a la universidad necesaria. IEC-Conadu

CRES. (2018). Declaración de la III Conferencia Regional de Educación Superior para América Latina y el Caribe. Integración Y Conocimiento, 7(2), 96-105

De La Cruz, C., y Sasia, P. (2008). La responsabilidad de la universidad en el proyecto de construcción de una sociedad. Obtenido de http://www.frcu.utn.edu.ar/ archivos/institucional/ planEstrategico/ LaResponsabilidadDeLaUniversidadEn ElProyectoDeConstrucci

Delgado, M., Vargas, J., y Ramos, D. (2008). Revista de Educación y Sociedad. IESALC/UNESCO

Delors, J., Al Mufti, I., Amagi, I., Carneiro, R., Chung, F., Geremek, B., Nanzhao, Z. (1996). La Educación encierra un tesoro, informe a la UNESCO de la Comisión Internacional sobre la Educación para el Siglo XXI (compendio). Comisión Internacional sobre la Educación para el Siglo XXI

Domínguez, J., y Rama, C. (2012). La Responsabilidad Social Universitaria en la Educación a Distancia. Universidad Católica Los Ángeles de Chimbote
Grimaldo, H. (2018). Colección Cres 2018. El rol de la educación superior de cara a los desafíos sociales de América Latina y el Caribe. Editorial Universidad Nacional de Córdoba

ISO. (2010). ISO 26000. Obtenido de https://www. iso.org/obp/ui\#iso:std:iso:26000:ed-1:v1:es

Martin, V. (2018). Calidad y responsabilidad social en la educación superior. En H. e. Grimaldo, El rol de la educación superior de cara a los desafíos sociales de América Latina y el Caribe (págs. 17-34). UNESCO-IESALC y UNC

ORSALC. (2016). Tendencias de la Responsabilidad Social Territorial (RST) 2012-2016. Observatorio de Responsabilidad Social para América Latina y el Caribe

Rama, C. (2018). Dinámicas de la educación superior a distancia y virtual en América Latina. Políticas, tensiones y tendencias de la educación y las tecnologías de comunicación. Obtenido de https://psychologynet.org/ sitemap/

Restrepo, R. (2013). Responsabilidad Social. Nueva Teoría-Nuevas Prácticas. ICONTEC Impresión Contacto Gráfico Ltda. Internacional

UNESCO. (1998). Declaración mundial sobre la Educación Superior en el siglo XXI: visión y acción. Obtenido de https://unesdoc.unesco. org/ark:/48223/pf0000113878_spa.locale=es

UNESCO. (2015). Transformar la Educación ¿hacia un bien común mundial? Obtenido de https://unesdoc.unesco.org/ark:/48223/ pf0000232697

UNESCO. (2019). Plan de Acción 20182028. Obtenido de http://www.iesalc. unesco.org/wp-content/uploads/2019/08/ PlandeAccionCRES2018-2028-Def.pdf

UNMSM. (2015). Modelo RSU San Marcos. Universidad Nacional Mayor de San Marcos

Valdés, H. (2008). Hacia una Evaluación del desarrollo y formación corporal, recional y emocional del ser humano. UNESCO 